\title{
Analysis and Forecasting of Electric Power Energy Consumption in IoT Environments
}

\author{
Vasanth Ragu ${ }^{1}$, Seung-Weon Yang ${ }^{2}$, Kangseok Chae ${ }^{3}$, Jangwoo Park ${ }^{4}$, \\ Changsun Shin ${ }^{5}$, Su Young Yang ${ }^{6}$ and Yongyun $\mathrm{Cho}^{7 *}$ \\ ${ }^{13457}$ Department of Information and Communication Engineering, Sunchon \\ National University, Suncheon-si, Republic of Korea - 57922 \\ ${ }^{1}$ vasanth4224@scnu.ac.kr, ${ }^{2}$ swyangy@gmail.com, ${ }^{3} 01056101111 @$ daum.net, \\ jwwpark@scnu.ac.kr, ${ }^{5}$ csshin@scnu.ac.kr, ${ }^{6}$ hi@elsys.kr, ${ }^{7} y y c h o @ s c n u . a c . k r$
}

\begin{abstract}
This study deals with analysis and forecast electric power energy consumption data considering socio-economic and demographical variables (gross domestic product $G D P$, gross national income - GNI, Population - PP) through linear regression model and artificial neural network (ANN) model. The main purpose of this study is to predict the accuracy of energy consumption neither overestimation nor underestimation. To do this, the suggested study correlates socioeconomic and demographic variables (GDP, GNI, \& PP) for energy consumption. Then, this study suggests four various models which consist of different combination of the variables. Through experiments, the suggested study compared and analyzed neural network models with linear regression models for the performance in energy consumption forecasting. Finally, this study suggests the best model for energy consumption forecasting in the result and conclusion section.
\end{abstract}

Keywords: Energy Consumption, GDP, GNI, Population, and IoT

\section{Introduction}

Presently, IoT technologies are rapidly growing in worldwide. The main part in a technology development is to utilize electric power energy and consider for all the electrical devices and machineries, which are utilizing electric power energy. The electric power energy is one of the vital roles for developing and developed countries in worldwide. As populations are increased, more requirements for energy consumption are also increased. Therefore, energy production and energy consumption are parts of important problems, which many countries in worldwide have to resolve.

In this research, we used historical data of Energy Consumption(EC) with socioeconomic and demographic variables, which consist of Gross Domestic Product (GDP), Gross National Income (GNI), and Population (PP) which are used to correlated to each other. Based on the correlation output, we found the relationship among the variables. The variables are very useful to predict accurately energy consumption and to avoid overestimation and underestimation of energy consumption. Afterward, we created four different models, each of which contains different combinations of variables. The Model 1 is designed to develop modelling and forecasting of energy consumption based on GDP and GNI. Model 2 aims to develop modelling and forecasting of energy consumption based on GDP and Population. Model 3 is designed to develop the modelling and forecasting of energy consumption based on GNI and Population. Finally, the purpose of Model 4 is to develop the modelling and forecasting of energy consumption depends on GDP, GNI, and Populaton.

Received (January 18, 2018), Review Result (April 10, 2018), Accepted (April 20, 2018)

* Corresponding Author 
The two types of forecasting techniques for this work are linear regression model (LM) and artificial neural network (ANN) model. Both forecasting models are implemented in four different models, which include a different combination of variables to forecast energy consumption. The linear model may be used as one of the most reasonable models in energy consumption forecasting, and the neural network may have advantages in aspect of computational speed and forecasting performance with fast processing times. The main purpose of this study is modelling and forecasting of energy consumption using GDP, GNI, and PP adopted with linear regression model and artificial neural network model. The result and conclusion of this study selects reasonable formulations to estimate energy consumption, picks out more potential models among those and then suggests the best forecasting model in energy consumption.

\section{Related Works}

A. Azadeh and et al., published a simulated based neural network algorithm for forecasting electrical energy consumption in $\operatorname{Iran[1].~F.~Egelioglu~and~et~al.,~}$ analysed the economic variables and electricity consumption in Northern Cyprus[2]. Vincenzo Bianco and et al., conducted a forecast research for Italy electricity consumption using linear regression models[3]. A. Azadeh and et al., analyzed and forecasting electricity consumption in industrial sectors by using neural network model [4]. Gamze Ogcu and et al., had published forecasting electricity consumption with neural networks and support vector regression [5]. Eva GonzalezRomera and et al. investigated forecasting of the electric energy demand trend and monthly fluctuation with neural networks[6]. A. Azadeh and et al., examined the forecasting electrical consumption by integration of neural network, time series and ANOVA [7]. Vincenzo Bianco and et al., expressed and forecasted of nonresidential electricity consumption in Romania [8]. Zaid Mohamed and Pat Bodger analyzed and forecasted electricity consumption in New Zealand using economic and demographic variables (GDP, the average price of electricity and Population) [9]. A. Azadeh and et al., investigated and predicted electrical energy consumption using artificial neural networks and genetic algorithm [10]. R. E. Abdel-Aal had published univariate modelling and forecasting method of monthly energy demand using time series and neural networks[11]. Kadir Kavaklioglu analysed energy consumption with socioeconomic and demographic variables (GNP, Population, Import, and Exports), then researched modelling and prediction of energy consumption in Turkey by using support vector regression [12]. Hsiao-Tien Pao investigated for Taiwan's electricity consumption using four economic factors (National Income, Population, GDP and Consumer Price Index) by comparing linear and nonlinear forecasts[13]. Zong Woo Geem and William E. Roper were investigated energy demand estimation of South Korea using four independent variables (GDP, Population, Import, and Exports) adopted with the artificial neural network [14]. A. Sozen and E. Arcaklioglu have developed the future projections of the basic energy sources in Turkey by using different independent variables (population, gross generation, installed capacity, net energy consumption, import, and exports) [15]. Adnan Sozen and et al., examined and forecasted Turkey's net energy consumption using four factors (Population, gross generation, installed capacity and years) [16]. Adan sozen and Erol Arcaklioglu were examined prediction of net energy consumption based on economic indicators (GDP, Gross National Product-GNP, Population)[17]. Adnan Sozen and et al., analyzed and forecasted net energy consumption using different factors (Population, gross generation, installed capacity and years) adopted neural network model[18]. So, based on the above reference this study suggests a method for forecasting energy 
consumption with socioeconomic and demographic variables (GDP, GNI, Population) using linear model and neural network model.

\subsection{Linear Regression Model}

Linear regression is defined as a relationship between a scalar dependent variable $\mathrm{Y}$ and one or more explanatory variables, which are called as independent variables, denoted as X [19]. If the one explanatory variable is presented, then it is called as a simple linear regression. But, if many explanatory variables are given, it is called as multiple linear regression [19]. When $X$ and $Y$ values are simultaneously increasing or decreasing which seem to be linear regression, but they are not straight lines. This model is the most popular basic type of regression and commonly used for predictive analysis [20]. The following linear regression equation is

$$
\hat{Y}=b X+c
$$

Where, $\hat{Y}$ - Estimated Variable or Dependent Variable,

$X$ - Independent Variable or Explanatory Variable,

$b$ - regression coefficient or slope of the line,

$c$-intercept value.

\subsection{Artificial Neural Network Model}

The human brain is a good example of an artificial neural network if we understand about the brain and its function then we can easily understand the artificial neural network. The brain has a large number of neurons, which are interconnected with each other. Similarly, ANN is a large number of computers (or nodes) and interconnected with each other. Because human's brain consists of 100 billion neurons, which are interconnected to each other, both brain and ANN are very similar in working, but not same. An ANN is a computational model based on the structure and functions of biological neural networks [21]. Information that flows through the network affects the structure of the ANN because a neural network changes - or learns, in a sense - based on that input and output [21]. ANN or connectionist systems are a computational model used in machine learning, computer science and other research disciplines [22]. ANN has been used to solve a wide variety of tasks, including computer vision, speech recognition, machine translation and medical diagnosis [22].

Figure 1 diagram suggests an easily understandable concept for the function of artificial neurons. In Figure 1, neurons are working through feedforward input data and backward error propagation. They are easily communicating from one to each other by using signals and weighted connections. The transfer function is used to estimate the summation of weighted inputs and then is converted to output. In this process, we used unit steps transfer function shows in Figure 2 (b). In unit steps, the output is set at one of two levels, based on whether the total input is greater than or less than some threshold value [23]. In this ANN model, we have used multi-layer perceptron with the back-propagation algorithm. The multi-layer perceptron(MLP) is a feedforward artificial neural network model that maps sets of input data onto a set of appropriate output [24]. An MLP consists of multiple layers of nodes in a directed graph, with each layer fully connected to the next one [24]. 


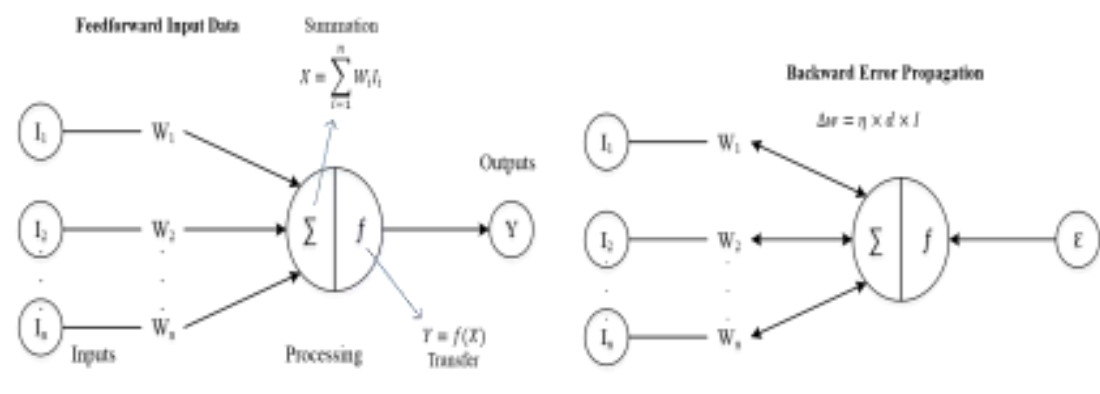

Figure 1. The Function of Artificial Neurons

Figure 2 (a) diagram shows the architecture of back-propagation network model. There are three layers, which consist of input layer the series of $\mathrm{I}_{\mathrm{n}}$, hidden layer the series of $\mathrm{H}_{\mathrm{m}}$, and output layer $Y_{k}$. The right side arrow is the direction of learning work and the left side arrow means the direction of error. Bias is defined as the difference between expected value and true value. In this case, the bias values always is 1 , which is the default value of the back-propagation neural network model.

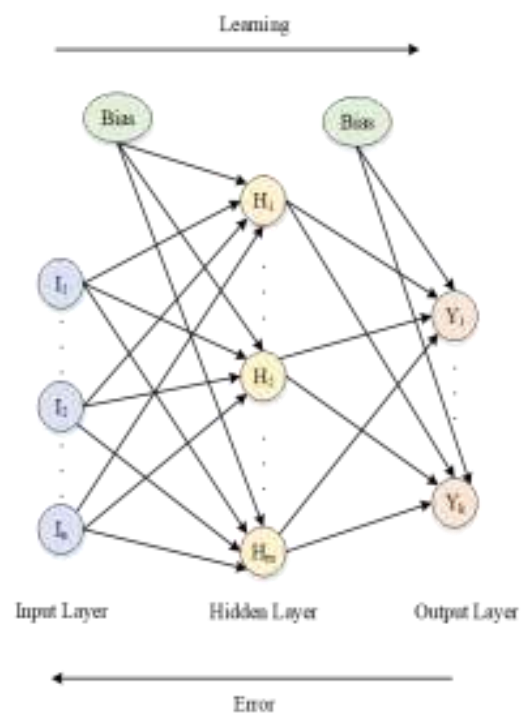

(a)

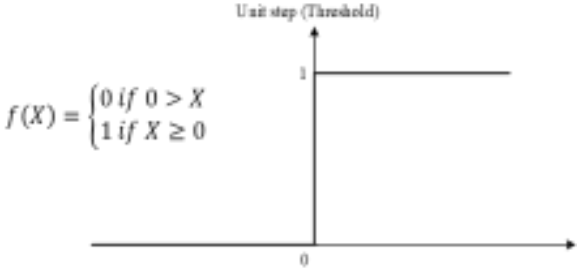

(b)

\section{Figure 2. (a) Diagram for Back-Propagation Network Model, (b) Diagram for Unit Steps (threshold) Transfer Function}

The input nodes are the values, which a user gives as inputs, and the hidden nodes are processing of input nodes. Finally, the output node provides a forecast for the future value. The model equation can be written as

$$
y_{t}=A_{0}+\sum_{j=1}^{n} A_{j} f\left(\sum_{i=1}^{m} B_{i j} y_{t-i}+B_{0 j}\right)+\varepsilon_{t}-r \text { ref }[1]
$$

Where, $m$ - number of input nodes,

$n$ - number of hidden nodes,

$f$ - unit step (threshold) transfer (activation) function,

$\left\{A_{j}, j=0,1_{s} \ldots n\right\}$ - weights (from hidden to output),

$\left\{B_{i j}, i=1,2, \ldots, m ; j=0,1, \ldots, n\right\}$ - weights (from input to hidden),

$A_{0}$ and $B_{0 j}$ - weights of arcs leading from bias terms. 


\subsection{Root Mean Square Error}

The root mean square error (RMSE) is also known as the root mean square deviation (RMSD). The RMSE is frequently used a measure of the difference between predicted values and actual observed values [25]. The following RMSE equation is,

$$
\text { RMSE }=\sqrt{\frac{\sum_{i=1}^{n}\left(\hat{y}_{\mathrm{i}}-\widehat{\left.\hat{N}_{i}\right)^{2}}\right.}{n}} \ldots \operatorname{ref}[25]
$$

Where, $y_{i}$ - actual observed values, $\hat{y}_{i}-$ predicted values by the model, $n$ - number of observation.

\subsection{Mean Absolute Error}

The mean absolute error (MAE) is an average of all absolute errors. In other words, the MAE is a quantity used to measure how close forecasts or predictions are to the eventual outcomes [26]. The MAE formula is

$$
M A E=\frac{1}{n} \sum_{i=1}^{n}\left|y_{i}-\widehat{y}_{i}\right| \ldots \text {.ref[26] }
$$

Where, $y_{i}-$ actual value, $\hat{y}_{i}-$ predicted value, and $n$ - number of observation.

\subsection{Mean Absolute Percentage Error}

The MAPE is a measure of prediction accuracy of a forecasting method in statistics [27]. The MAPE is also known as mean absolute percentage deviation (MAPD) [27]. The following formula of MAPE is,

$$
\text { MAPE }=\frac{100}{n} \sum_{i=1}^{n}\left|\frac{y_{i}-\hat{y}_{i}}{y_{i}}\right|_{\ldots \operatorname{ref}[27]}
$$

Where, $y_{i}-$ actual value, $\hat{y}_{i}-$ forecast value, $n-$ number of iterations.

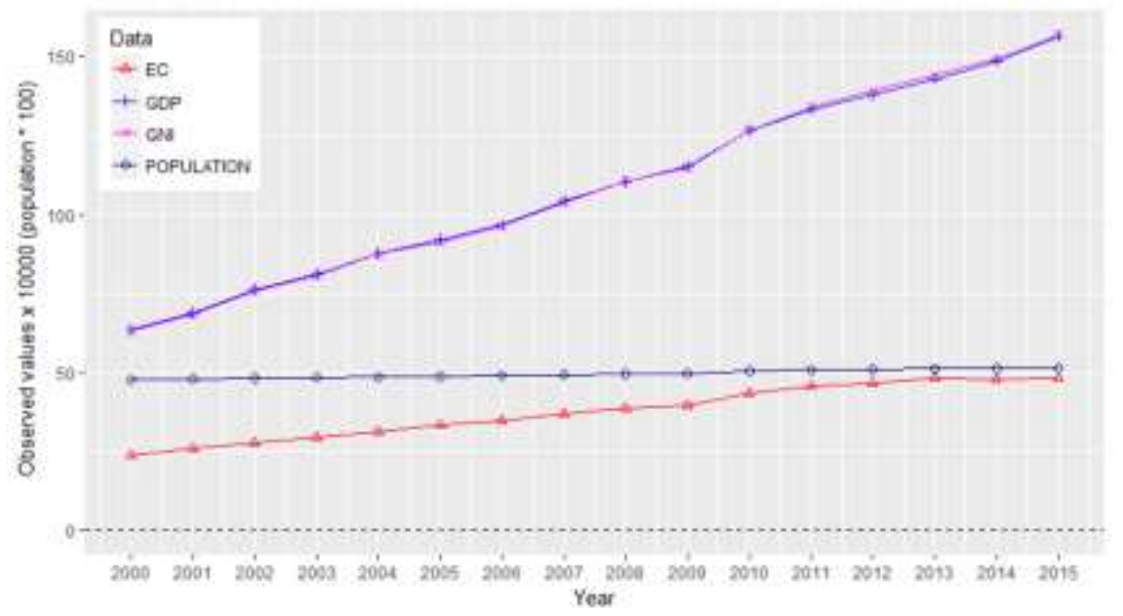

Figure 3. Energy Consumption with Three Independent Variables

\section{Materials and Methods}

In this section, we are used three independent variables namely GDP, GNI and Population along with energy consumption. A measure of overall economic output in a country which is called gross domestic product and the GNI is a total value that is produced within a country, which comprises of the gross domestic product along with the income obtained from other countries (dividends, interests) [28]. Both GDP and GNI are opposite side of the same coin. The population is one of the most important independent 
variables because when the population increased and then companies and manufacturing products also increased with corresponding economic factors (GDP and GNI) are increased along with more energy consumption.

The historical data (from 2000 to 2015) of socio-economic and demographic variable along with energy consumption is plotted in figure 3 . The red, navy, blue and pink colors and then the corresponding factors are energy consumption, population, GDP, and GNI. The unit of energy consumption is gigawatt hour.

\subsection{Pearson Correlation Coefficient}

Pearson Correlation Coefficient (PCC) is a measure of the linear correlation between two variables $X$ and $Y$ [29]. It has a value between +1 and -1 , where 1 is a total positive correlation, -1 is a total negative correlation, and 0 is no correlation [29]. The formula of Pearson correlation coefficient $(\rho)$ is,

$$
\operatorname{cor}(X, Y)=\rho=\frac{\operatorname{Cov}(X, Y)}{\sigma_{X} \sigma_{Y}} \ldots \operatorname{ref}[29]
$$

Where, $\operatorname{cov}\left(X_{x} Y\right)=E\left[\left(X-\mu_{X}\right)\left(Y-\mu_{Y}\right)\right], \mathrm{E}-$ Expectation, Cov - Covariance, $\mu_{X}-$ mean value of $\mathrm{X}, \mu_{Y}-$ mean value of $\mathrm{Y}, \sigma_{X}-$ standard deviation of $\mathrm{X}, \sigma_{Y}-$ standard deviation of $\mathrm{Y}$.

Using Pearson correlation coefficient, we can find the relationship between energy consumption, GDP, GNI, and Population. The main objective of the correlation is to check relationship accuracy of energy consumption. If the correlation value is 1 or close to 1 , then we can easily identify the precision of relationship is high in energy consumption. Figure 4 shows that the result of the correlation coefficient for Energy consumption (EC), GDP, GNI, and Population (PP). In this result, red, white, and blue refers to positive correlation, no correlation, and negative correlation respectively. The results of Pearson correlation are shown with the correlated variable boxes shown with red colors, which mean that those are highly positive and perfectly correlated with each other.

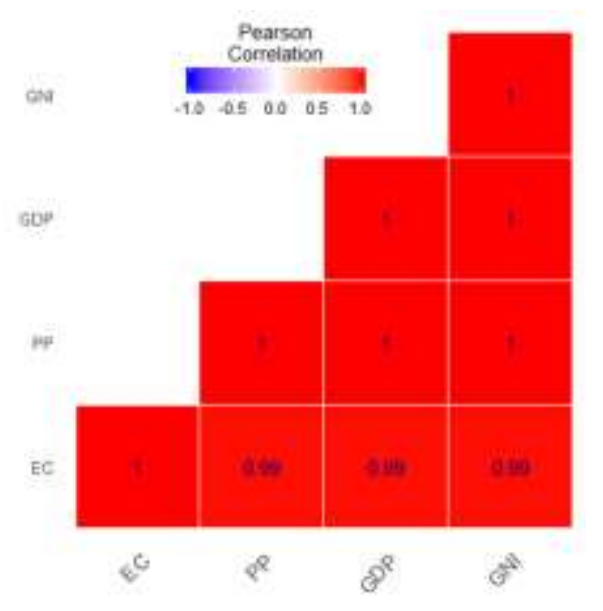

Figure 4. The Result of Pearson Correlation Coefficient

\subsection{Multiple Linear Regression}

The multiple linear regression can be implemented in four different combinations of independent variables of models. The following equations are

Model 1: $y=a_{1} x_{1}+b_{1} x_{2}+f$

Model 2: $y=a_{2} x_{1}+b_{2} x_{2}+f$ 
Model 3: $y=a_{a} x_{2}+b_{a} x_{a}+f$

Model 4: $y=a_{4} x_{1}+b_{4} x_{2}+c_{4} x_{3}+f$

Where, $y$-estimated energy consumption,

$x_{1}-$ Gross Domestic Product,

$x_{2}$ - Gross National Income,

$x_{a}-$ Population,

$\{$ the series of $a, b \& c\}$ - regression coefficient, $f$-intercept values.

The four models of multiple linear regression equations can be estimated in Table 1 . The historical data (from 2000 to 2015) is divided into two parts, a training data set and a testing data set. Using regression model, testing data can estimate the regression coefficient and $\mathrm{R}^{2}$ values. The $\mathrm{R}^{2}$ values of regression models are $0.9878,0.9872,0.9875$, \& 0.9878 and the corresponding Model 1, 2, 3, \& 4, respectively. Using mean absolute percentage error (MAPE) value, Model 4 is the best fit in the linear model. How because the MAPE value of Model 4 is less when compared with others (Table 3). Therefore, Model 4 is best fit in a multiple linear regression model and their MAPE value is 0.3377 .

Table 1. Estimated Coefficient Value of a Multiple Linear Regression and $\mathbf{R}^{2}$ Value

\begin{tabular}{llllll}
\hline & GDP & GNI & PP & \multicolumn{1}{c}{ Intercept } & \multicolumn{1}{c}{$\mathrm{R}^{2}$} \\
\hline Model 1 & -0.5313 & 0.8010 & - & 81617.71 & 0.9878 \\
Model 2 & 0.19982398 & - & 0.01926914 & -797566.96 & 0.9872 \\
Model 3 & & 0.22554341 & 0.01245035 & -487501.53 & 0.9875 \\
Model 4 & -0.491519073 & 0.749121614 & 0.003006487 & -54302.86 & 0.9878 \\
\hline
\end{tabular}

\subsection{Implemented Artificial Neural Network Model}

In this study, ANN is one of the most important models to analyze and forecast of energy consumption with socio-economic and demographic variables (GDP, GNI, \& Population). The ANN techniques are implemented in four different models and we get outputs of energy consumption. The historical data can be split into two parts training set and testing set. The training set is used to learning the purpose and the testing set is used to involve estimate and forecast the energy consumption. In a neural network, it is crucial to choose the number of hidden layers. If the size is too small or big, then the error values do not decrease. So, we choose proper size of hidden layers. Figure 5 shows the plotted neural network of four models. The middle two layers are hidden layers, and first and last layers are individually an input and an output layers, respectively. The bias is connected with hidden layers and an output layer. The input layer consists of various types of independent variables, which are GDP, GNI \& Population. The output layer provides forecasting of energy consumption.

Table 2 shows the result of ANN models, the estimated values of root mean square error and the mean absolute error. In Table 2, each of models consists of input layers, hidden layers, an reached threshold value, the number of steps, an error value, RMSE and MAE. The number of input node is 2 or 3 , and the number set of hidden nodes is $(5,3)$. The numeric value specifies the threshold for the partial derivatives of the error function as a stopping criteria [30]. The default value of the threshold is 0.01 . Table 2 shows that the reached threshold values are less than 0.01 . The error value refers to the likelihood of calculating Akaike's Information Criterion. Using RMSE and MAE, the overall performance of error in this model can be found. 

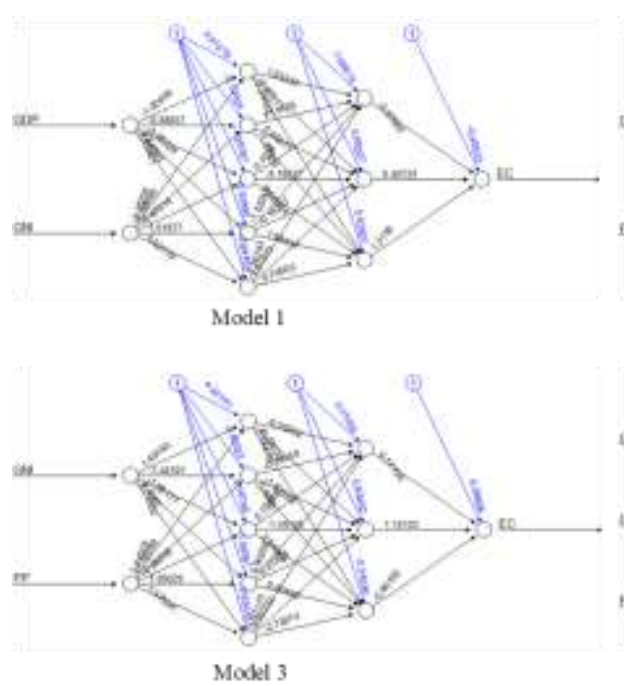

Figure 5. The Four Models of Neural Network

Table 2. The Result of ANN Model and Estimated the RMSE \& MAE

\begin{tabular}{cccccccc}
\hline & $\begin{array}{c}\text { Input } \\
\text { Layer }\end{array}$ & $\begin{array}{l}\text { Hidden } \\
\text { Layers }\end{array}$ & $\begin{array}{l}\text { Reached } \\
\text { Threshold }\end{array}$ & $\begin{array}{l}\text { Number } \\
\text { of steps }\end{array}$ & Error & RMSE & MAE \\
\hline Model 1 & 2 & $(5,3)$ & 0.008696 & 77 & 0.005038 & 6420.87 & 4959.94 \\
Model 2 & 2 & $(5,3)$ & 0.009782 & 39 & 0.002912 & 5363.78 & 3997.94 \\
Model 3 & 2 & $(5,3)$ & 0.005315 & 85 & 0.003217 & 5469.72 & 3880.86 \\
Model 4 & 3 & $(5,3)$ & 0.008694 & 46 & 0.002671 & 5179.08 & 3607.47 \\
\hline
\end{tabular}

Note: RMSE - Root Mean Square Error, MAE - Mean Absolune Erroe

Based on the result of ANN, Model 4 is best fit in comparison with the remaining other models. The error value of Model 4 is very less, and the overall performance of error in RMSE \& MAE values is lower than one of others. Therefore, in this study, the values of the Model 4 for error, RMSE and MAE as the corresponding factors are 0.002671, $5179.08,3607.47$ respectively.

\section{Result and Discussion}

In this section, four models were used to determine the forecasted energy consumption $(\mathrm{GWh})$ using socioeconomic and demographic variables and real data in comparison with regression and neural network. With test datasets from 2009 to 2015, we estimated and forecasted amount of energy consumption using the linear model and neural network. The result of Model 1, 2, 3, \& 4 were shown in Figures 6, 7, 8, \& 9 respectively. 


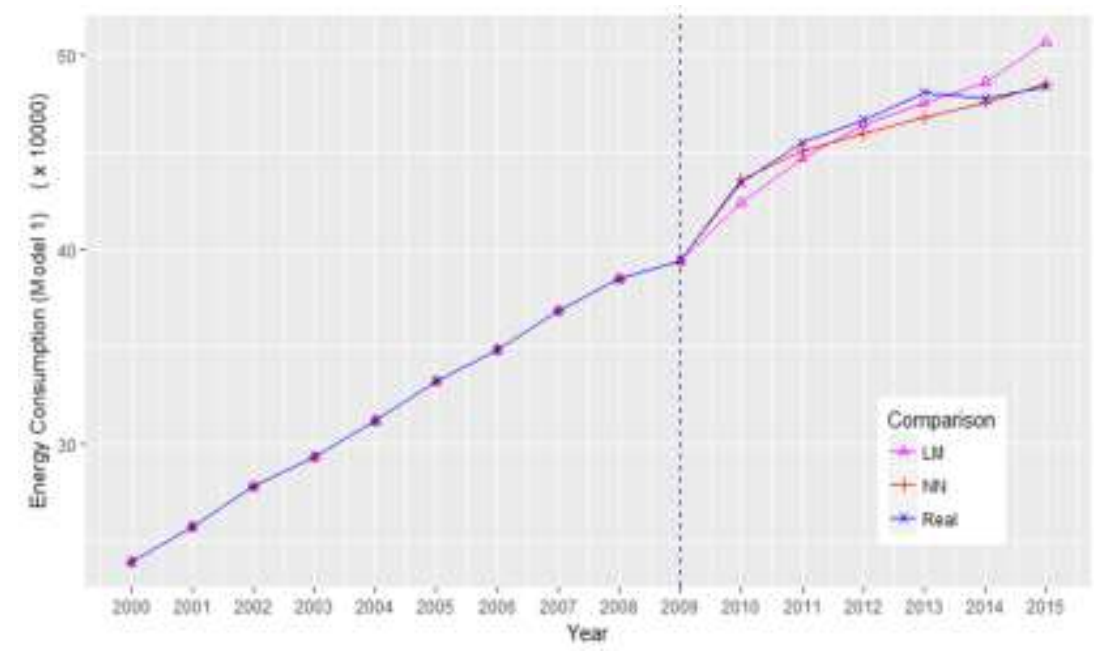

Figure 6. The Result of Model 1 by Comparing Real Data, Linear Model, and Neural Network

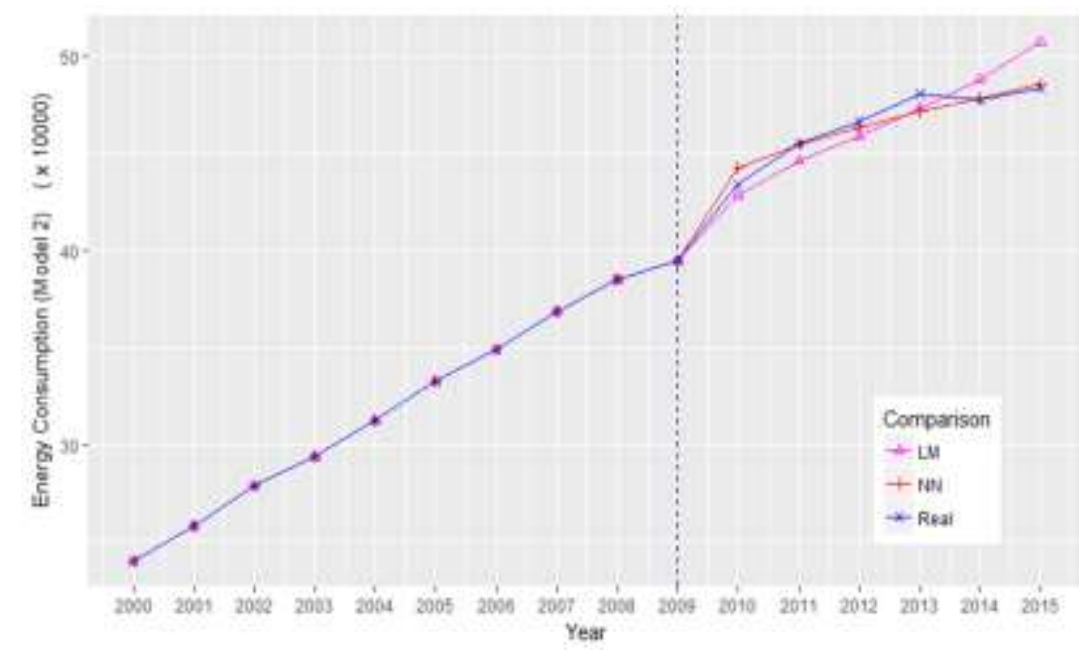

Figure 7. The Result of Model 2 by Comparing the Real Data, Linear Model, and Neural Network

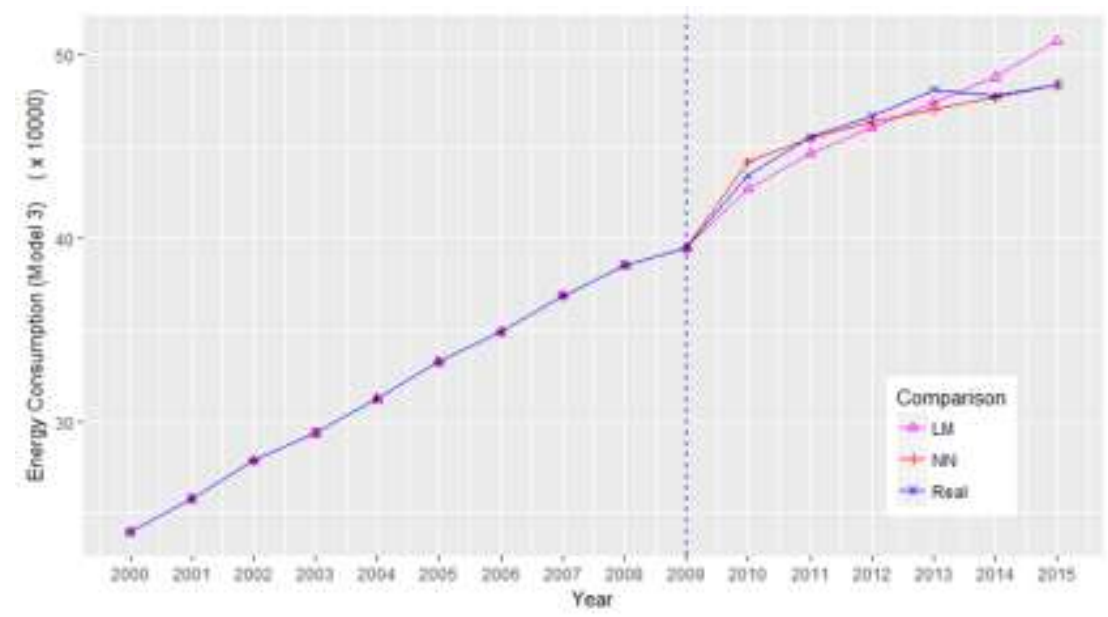

Figure 8. The Result of Model 3 by Comparing the Real Data, Linear Model, and Neural Network 


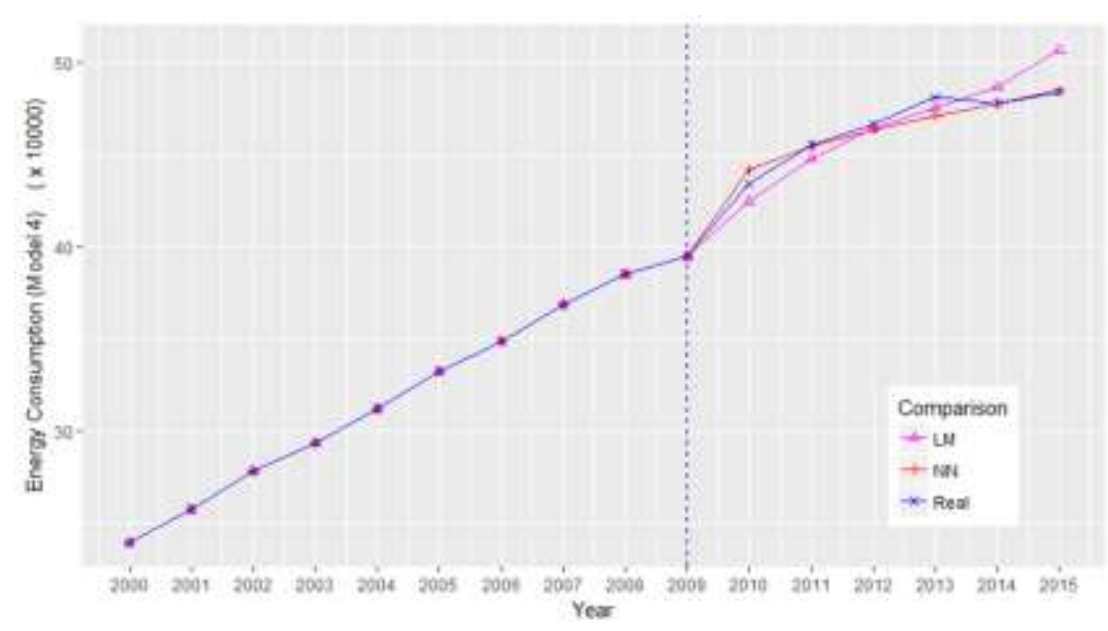

Figure 9. The Result of Model 4 by Comparing the Real Data, Linear Model, and Neural Network

In general, AIC and BIC can be useful to find and select a true model. To do that, we found AIC and BIC for calculated system load performance between comparing linear model and neural network. Table 3 shows result values, which consist of an estimated mean absolute percentage error, Akaike's Information Criteria (AIC), Bayesian Information Criteria (BIC) through a comparing test the linear model with neural network.

Table 3. Estimated MAPE, AIC \& BIC in Comparison with Linear Model and ANN

\begin{tabular}{|c|c|c|c|c|}
\hline & & \multirow[b]{2}{*}{ MAPE } & \multicolumn{2}{|c|}{ System Load Performance } \\
\hline & & & AIC & BIC \\
\hline \multirow{4}{*}{$\begin{array}{l}\text { Linear } \\
\text { Model }\end{array}$} & Model 1 & 0.3411 & 344.75 & 347.83 \\
\hline & Model 2 & 0.3758 & 345.59 & 348.68 \\
\hline & Model 3 & 0.3704 & 345.17 & 348.26 \\
\hline & Model 4 & 0.3377 & 346.74 & 350.60 \\
\hline \multirow{4}{*}{$\begin{array}{l}\text { Neural } \\
\text { Netwotk }\end{array}$} & Model 1 & 0.1757 & 74.01 & 102.59 \\
\hline & Model 2 & 0.1444 & 74.01 & 102.59 \\
\hline & Model 3 & 0.1399 & 74.01 & 102.59 \\
\hline & Model 4 & 0.1302 & 84,00 & 116.45 \\
\hline
\end{tabular}

The AIC and BIC values of the neural network model are very lower than those of the linear model, and the mean absolute percentage error of it values were also very low. The MAPE values of neural network are $0.1757,0.1444,0.1399$ and 0.1302 with the Model 1 , $2,3, \& 4$, respectively. Therefore, artificial neural network model can be a best-fit model for forecast in energy consumption.

\section{Conclusion}

The main objective of this research is to predict the accuracy of energy consumption using GDP, GNI, and Population in comparison with linear model and neural network. To do that, we suggested 4 reasonable models considering socioeconomic and demographic variables as. On the result of multiple linear regression and neural network techniques, the RMSE, MAE, and MAPE are very less when are compared with those of others. Through the test, we proved that the suggested Model 4, which uses GDP GNI, and 
Population as the combination of independent variables, is best fitted for forecasting energy consumption. In the result and discussion, we suggested that the estimated MAPE, AIC and BIC values of neural network model are very lower than those values of linear model. And so, we found that the neural network is best forecast model in energy consumption.

Finally, we concluded that the Model 4 is best-fitted model through the result values of MAPE, which are 0.3377 in LM and 0.1302 in NN. And, we also know that ANN is bestforecasted techniques through the results values of MAPE, which are 0.17 in Model 1 and 0.14 in Model 2, 0.14 in Model 3, and 0.13 in Model 4 for Korea electric power energy consumption data.

\section{Acknowledgments}

This work was supported by the Korea Institute of Energy Technology Evaluation and Planning(KETEP) and the Ministry of Trade, Industry \& Energy(MOTIE) of the Republic of Korea (No. 20172010000730).

This paper is a revised and expanded version of a paper entitled "A Best Fit Model for Forecasting Korea Electric Power Energy Consumption in IoT Environments" presented at 2017 International Interdisciplinary Workshop, Daejeon University, 21-23 ${ }^{\text {rd }}$ December 2017.

\section{References}

[1] A. Azadeh, S. F. Ghaderi and S. Soharbkhani, "A simulated-based neural network algorithm for forecasting electrical energy consumption in Iran", Energy Policy, vol. 36, no. 7, (2008), pp. 2637-2644.

[2] F. Egelioglu, A. A. Mohamad and H. Guven, "Economic variables and electricity consumption in Northern Cyprus", Energy, vol. 26, no. 4, (2001), pp. 355-362.

[3] V. Bianco, O. Manca and S. Nardini, "Electricity consumption forecasting in Italy using linear regression models", Energy, vol. 34, no. 9, (2009), pp. 1413-21.

[4] A. Azadeh, S. F. Ghaderi and S. Sohrabkhani, "Annual electricity consumption forecasting by neural network in high energy consuming industrial sectors", Energy Conversion and Management, vol. 49, no. 8, (2008) August, pp. 2272-78.

[5] G. Ogcu, O. F. Demirel and S. Zaim, "Forecasting Electricity Consumption with Neural Networks and Support Vector Regression", 8th International Strategic Management Conference, Turkey, (2012), pp. 1576-85.

[6] E. Gonzalez-Romera, M. Angel Jaramillo-Moran and D. Carmona-Fernandez, "Forecasting of the electric energy demand trend and monthly fluctuation with neural networks", Computer \& Industrial Engineering, vol. 52, no. 3, (2007), pp. 336-343.

[7] A. Azadeh, S. F. Ghaderi and S. Sohrabkhani, "Forecasting electrical consumption by integration of neural network, time series and ANOVA", Applied Mathematics and Computation, vol. 186, no. 2, (2007), pp. 1753-1761.

[8] V. Bianco, O. Manca, S. Nardini and A. A. Minea, "Analysis and forecasting of nonresidential electricity consumption in Romania", Applied Energy, vol. 87, no. 11, (2010), pp. 3584-3590.

[9] Z. Mohamed and P. Bodger, "Forecasting electricity consumption in New Zealand using economic and demographic variables", Energy, vol. 30, no. 10, (2005), pp. 1833-43.

[10] A. Azadeh, S. F. Ghaderi, S. Tarverdian and M. Saberi, "Integration of artificial neural networks and genetic algorithm to predict electrical energy consumption", Applied Mathematics and Computation, vol. 186, no. 2, (2007), pp. 1731-1741.

[11] R. E. Abdel-Aal, "Univariate Modeling and forecasting of monthly energy demand time series using abductive and neural networks", Computers \& Industrial Engineering, vol. 54, no. 4, (2008), pp. 903917.

[12] K. Kavaklioglu, "Modeling and prediction of Turkey's electricity consumption using Support Vector Regression", Applied Energy, vol. 88, no. 1, (2011), pp. 368-375.

[13] H.-T. Pao, "Comparing linear and nonlinear forecasts for Taiwan's electricity consumption", Energy, vol. 31, no. 12, (2006), pp. 2129-2141.

[14] Z. Woo Geem and W. E. Roper, "Energy demand estimation of South Korea using artificial neural network", Energy Policy, vol. 37, no. 10, (2009), pp. 4049-4054.

[15] A. Sozen and E. Arcaklioglu, "Prospects for Future Projections of the Basic Energy Sources in Turkey", Energy Sources, Part B: Economics, Planning, and Policy, vol. 2, no. 2, (2007), pp. 183-201.

[16] A. Sozen, E. Arcaklioglu and M. Ozkaymak, “Turkey's net energy consumption”, Applied Energy, vol. 81, no. 2, (2005), pp. 209-221. 
[17] A. Sozen and E. Arcaklioglu, "Prediction of net energy consumption based on economic indicators (GNP and GDP) in Turkey", Energy Policy, vol. 35, no. 10, (2007), pp. 4981-4992.

[18] A. Sozen, M. Ali Akcayol and E. Arcaklioglu, "Forecasting Net Energy Consumption using Artificial Neural Network", Energy Source, Part B: Economics, Planning, and Policy, vol. 1, no. 2, (2006), pp. 147-155.

[19] Linear Regression from Wikipedia, https://en.wikipedia.org/wiki/Linear_regression.

[20] Linear Regression from Statistics Solutions, http://www.statisticssolutions.com/what-is-linearregression/.

[21] Artificial Neural Network from Techopedia, https://www.techopedia.com/definition/5967/artificialneural-network-ann.

[22] Artificial Neural Network from Wikipedia, https://en.wikipedia.org/wiki/Artificial_neural_network.

[23] Artificial Neural Network from website, http://www.saedsayad.com/artificial_neural_network.htm.

[24] Multilayer Perceptron from Wikipedia, https://en.wikipedia.org/wiki/Multilayer_perceptron.

[25] Root Mean Square Error from Wikipedia, https://en.wikipedia.org/wiki/Root-mean-square_deviation.

[26] Mean Absolute Error from, https://www.kaggle.com/wiki/MeanAbsoluteError.

[27] Mean Absolute Percentage from https://en.wikipedia.org/wiki/Mean_absolute_percentage_error.

[28] Difference Between GNI and GDP from, http://www.differencebetween.net/business/differencebetween-gni-and-gdp/.

[29] Pearson correlation coefficient from https://en.wikipedia.org/wiki/Pearson_correlation_coefficient.

[30] F. Gunther and S. Fritsch, "neuralnet: Training of Neural Networks", The R journal, vol. 2, no. 1, (2010).

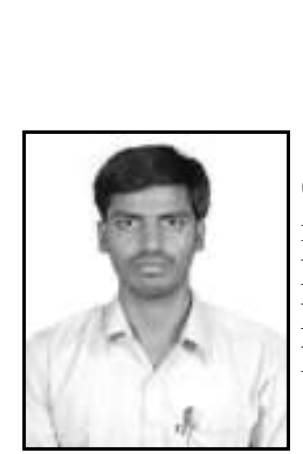

\section{Authors}

Vasanth Ragu, he completed Master of Computer Application (MCA) degree from India. He is pursuing a Doctorate degree in information and Communication Engineering at Sunchon National University in South Korea. His area of interest includes Forecast Model, Ubiquitous Computing, and Big Data Processing. Email: vasanth4224@scnu.ac.kr

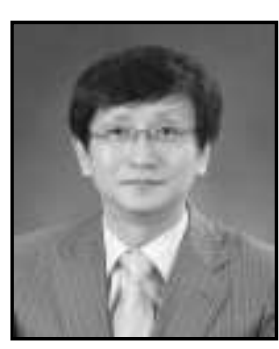

Seung-Weon Yang, he completed Doctorate degree in Computer Engineering at Jeonbuk National University in South Korea. He is a professor of computer engineering at Woosuk University. His area of interest includes Big Data Processing, Natural Language Processing, and AI algorithms. Email: swyangy@ gmail.com

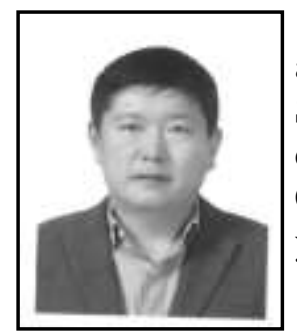

Kangseok Chae, he received Bachelor degree on the Information and Communication Engineering at Sunchon National University in South Korea and currently pursuing Master degree in same department. His area of interest Ontology Model, Ubiquitous Computing and Big data processing. He has worked as CEO for 15 years in the IT company in Korea. Email: 01056101111@daum.net 


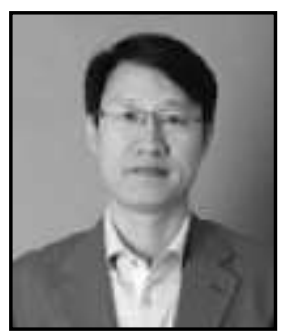

Jangwoo Park, he received the $\mathrm{BS}$, MS and $\mathrm{PhD}$ degrees in Electronic engineering from Hanyang University, Seoul, Korea in 1987, 1989 and 1993, respectively. In 1995, he joined the faculty member of the Sunchon National University, where he is currently a professor in the Department of Information \& Communication engineering. His research focuses on Localization and $\mathrm{SoC}$ and system designs and RFID/USN technologies. Email: jwpark@scnu.ac.kr

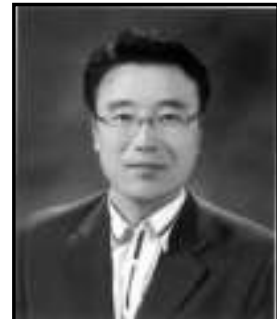

Changsun Shin, he received the $\mathrm{PhD}$ degree in computer engineering at Wonkwang University. Currently, he is an associate professor of the Department of information \& communication engineering in Sunchon National University. His researching interests include Distributed Real-Time Computing, Distributed Object Modeling, Ubiquitous Agriculture and Ubiquitous Sensor Network (USN).Email: csshin@ @scnu.ac.kr

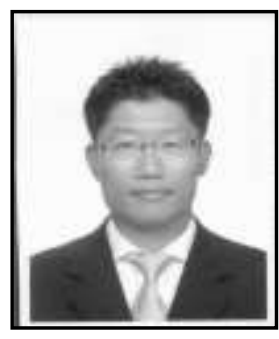

Su Young Yang, he is the CEO of ELSYS Corporation. He received Master degree on Computer Science in South Korea. And currently pursuing Doctorate degree in the Computer Science. His area of interest includes Advanced Agriculture Technology, IT Convergence, Cloud and Ubiquitous Computing. Email: hi@elsys.kr

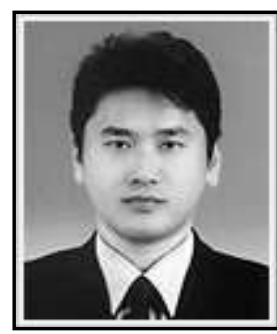

Yongyun Cho, he received the $\mathrm{PhD}$ degree in computer engineering at Soongsil University. Currently, he is an assistant professor of the Department of Information \& communication engineering in Sunchon National University. His main research interests include System Software, Embedded Software and Ubiquitous Computing. Email: yycho@scnu.ac.kr 
International Journal of Grid and Distributed Computing

Vol. 11, No. 6 (2018) 